\title{
Fertilización Química y órgano-mineral del Pasto Mulato (Brachiaria Híbrido) y Xaraés (Brachiaria Brizantha Xaraés). Santo Domingo De Los Tsáchilas.
}

Leonardo Jácome Gómez Manuel Suquilanda $V_{{ }_{2}}$

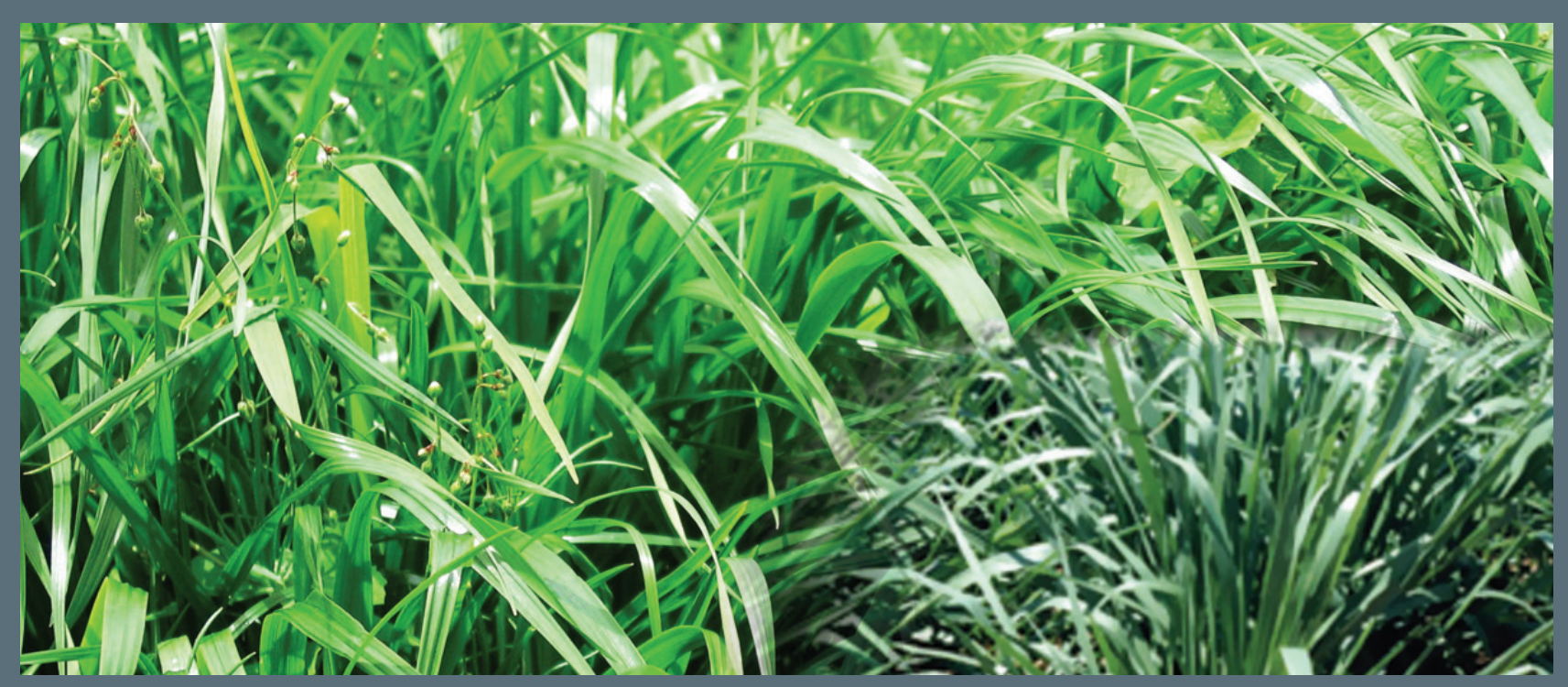

Resumen-La presente investigación se llevó a cabo en la Provincia de Santo Domingo de los Tsáchilas, Parroquia de Santo Domingo, Recinto Praderas del Toachi, en la Finca "La Maravilla", queestá ubicadaa $79^{\circ} 9^{\prime} 18^{\prime \prime}$ delongitud Oeste, $0^{\circ} 11^{\prime} 8^{\prime \prime}$ delatitud Sur y a una altitud de 465 msnm. El tema de trabajo de investigación fue "Fertilización química y órgano-mineral del pasto Mulato (Brachiaria híbrido) y Xaraés (Brachiaria brizantha Xaraés) - 2006".

El objetivo general fue determinar la respuesta de los pastos Mulato y Xaraés a la fertilización química y órgano-mineral, en las condiciones agroecológicas de Santo Domingo. Los objetivos específicos fueron: Establecer los mejores rendimientos y calidad de materia seca del pasto Mulato y Xaraés; determinar la fertilización más adecuada para los pastos Mulato y Xaraés y realizar un estudio económico de los tratamientos en estudio.

Se utilizó un Diseño de Bloques Completos al Azar, en arreglo factorial $2 \times 5$ y para el análisis de variancia de los cortes se empleó un diseño de parcela dividida con sub-parcelas con observaciones repetidas. Está investigación contó con cuatro repeticiones, con 10 tratamientos cada una, fruto de la interacción de los dos factores en estudio (Pastos Brachiarias x Fertilización). Se evaluaron siete variables: Cobertura (\%), Altura de Planta (cm), Producción de Materia Verde (t/ha/ año), Rendimiento de Materia Seca (t/ha/año), Incidencia de Malezas (\%), Calidad del forraje (Análisis Bromatológico y foliar) y un Análisis Económico de los tratamientos.

Se realizó la preparación del terreno (arada y rastrada), posteriormente se aplicó la fertilización inicial, un $40 \%$ antes de la siembra y el 60\% distribuido en los cortes, previo al respectivo análisis de suelo, con las siguientes cantidades (kg/ha/año): Fertilización 1: Nitrato de Amonio 476 (34\%), Superfosfato Triple 285 (46\%) (80\% al inicio), Muriato de Potasio 135 (60\%) y Sulfato de Magnesio 107 (25\%). Para el fertiforraje costa establecimiento (13-26-13-4-3) se empleó 329 kg/ha al inicio y el fertiforraje costa producción (21-12-15-4-3) 493 kg/ha/año en los cortes. Los fertilizantes orgánicos se incorporaron al voleo, un 60\% a la siembra y un $40 \%$ distribuido en los cortes de igualación. Del Vermicompost

1 Leonardo Jácome G. Egresado de la Maestría de Nutrición Vegetal. 099616361

2 Manuel Suquilanda V. Profesor de la cátedra de Abonos Orgánicos de la Universidad Tecnológica Equinoccial. (Director de tesis). 
bovino se empleó 24.7 t/ha y del Compost de estiércol bovino 26.6 t/ha. La siembra se realizó al voleo, empleándose 5 kg/ ha de semillas de pastos Mulato y Xaraés. El primer corte de igualación se efectuó a los 100 días después de la siembra, luego los cortes a los 30 días en invierno y 40 días en verano.

La investigación permitió establecer las siguientes conclusiones: El mejor rendimiento de materia seca, la obtuvo el pasto Mulato (Brachiaria hibrido) con 35.0 t/ha/año, seguido por el pasto Xaraés (Brachiaria brizantha Xaraés) con 33.8 t/ha/año, sometidos en ambos casos a la fertilización 1. La concentración más alta de proteína con 20.8\% del análisis bromatológico y los mejores niveles de contenido de nutrientes en el análisis foliar lo presentó el T2 (Mulato con fertilización 1). La Tasa de Acumulación de Materia Seca más alta la consiguió el T9 (Xaraés con vermicompost) con 19.2\%. El mejor porcentaje de cobertura de Brachiaria se observó en el T2 (Mulato con fertilización 1) con 85.6\%. La mayor altura de pasto Xaraés se obtuvo con el T7 (Xaraés con fertilización 1) con $99.6 \mathrm{~cm}$ y del pasto Mulato en el T3 (Mulato con fertiforraje) con $85.1 \mathrm{~cm}$. Los suelos sometidos a la fertilización orgánica resultaron mejorados significativamente en términos porcentuales de materia orgánica y provisión de nutrimentos (nitrógeno, calcio, magnesio y boro) al final del ensayo. Desde el punto de vista económico, con el T7 (Xaraés con fertilización 1), se consiguió el más alto beneficio neto con 388 dólares/ha/año, seguido por el T2 (Mulato con fertilización 1) con 367 dólares/ha/año. La mejor Tasa de Retorno Marginal la presentó el T7 (Xaraés con fertilización 1) con 46.6\%, la relación Beneficio/Costo más alta se la obtuvo con el T6 (Xaraés sin fertilización) con 1.44, seguida por el T8 (Xaraés con fertiforraje) con 1.40 con la que se obtiene mejor calidad del pasto.

De acuerdo a los resultados se formulan las siguientes recomendaciones, para las condiciones agroecológicas del área del ensayo: Fertilizar los pastos Mulato (Brachiaria hibrido) y Xaraés (Brachiaria brizantha Xaraés) con la Fertilización 1 (mezcla de fertilizantes químicos simples) en cantidad de 1026 kg/ha/año. Aplicar Compost o Vermicompost a los pastos Mulato (Brachiaria hibrido) y Xaraés (Brachiaria brizantha Xaraés) en cantidad de 26.6 t/ha y 24.7 t/ha, respectivamente, con una frecuencia de cada tres años. Para futuras investigaciones, asociar los pastos de este ensayo con leguminosas arbustivas para mejorar la calidad nutrimental del pastizal.

Palabras clave-Análisis, Fertilización, Pasto mulato, Xaraés.

\section{Introducción}

La disponibilidad comercial de gramíneas y leguminosas forrajeras tropicales ha aumentado en el Ecuador; sin embargo, continúa la búsqueda de más y mejores opciones forrajeras que respondan a la diversidad de la ganadería, así como también, la identificación de especies forrajeras de alta calidad, que toleren la incidencia de plagas que afectan la productividad y persistencia de los pastos. El Cantón Santo Domingo tiene 193776 ha, dedicada a ganadería bovina (pastizales), en las que existen 318540 Unidades Bovinas Adultas (UBA). Cuyos pastizales están constituidos por las especies de: Saboya, 98000 ha (50.5\%); Brachiaria, 52000 ha (26.8\%); Elefante, 21000 ha (10.8\%); Gramalote, 13276 ha (6.8\%); Micay, 3500 ha (1.8\%); Miel, 3000 ha (1.5\%); Estrella, 2500 ha (1.2\%) y Leguminosas Forrajeras, 500 ha (0.6\%) (MAG, 2003).

Se están introduciendo nuevas especies del género Brachiaria, con gran aceptación de los ganaderos de ésta zona, como son: El pasto Mulato (Brachiaria híbrido CIAT 36061), primer híbrido comercial obtenido por el Proyecto de Forrajes Tropicales del CIAT, destacado por la buena adaptación a un rango amplio de localidades, alta producción y calidad del forraje y la facilidad en el establecimiento por medio de semillas (AGRIPAC, 2004). El pasto Xaraés (Brachiaria brizantha Xaraés) evaluada por el EMBRAPA durante 15 años y liberada en el 2003; teniendo una alta productividad especialmente de hojas, un rápido rebrote y un florecimiento tardío (FERTISA, 2004).

\section{Materiales y Métodos}

La presente investigación se llevó a cabo en la Provincia de Santo Domingo de los Tsáchilas, Parroquia de Santo Domingo, Recinto Praderas del Toachi, en la Finca "La Maravilla", que está ubicada a $79^{\circ} 9^{\prime} 18^{\prime \prime}$ de longitud Oeste, $0^{\circ} 11^{\prime} 8^{\prime \prime}$ de latitud Sur y a una altitud de 465 msnm. Se utilizó un Diseño de Bloques Completos al Azar, en arreglo factorial 2x5 y para el análisis de variancia de los cortes se empleó un diseño de parcela dividida con sub-parcelas con observaciones repetidas. Está investigación contó con cuatro repeticiones, con 10 tratamientos cada una, fruto de la interacción de los dos factores en estudio: Pastos Brachiarias (p) x Fertilización (f). Los pastos evaluados fueron: p1 = Mulato y p2 = Xaraés; y los fertilizantes, $\mathrm{f0}=$ Sin fertilización (testigo), f1= Fertilización completa (mezcla fertilizantes simples), $\mathrm{f} 2=$ Fertiforraje Costa (fórmula compuesta), f3= Vermicompost y $\mathrm{f} 4=$ Compost Bovino. Se evaluaron siete variables: Cobertura (\%), Altura de Planta (cm), Producción de Materia Verde (t/ha/ año), Rendimiento de Materia Seca (t/ha/año), Incidencia de Malezas (\%), Calidad del forraje (Análisis Bromatológico y foliar) y un Análisis Económico de los tratamientos.

\subsection{Manejo del Experimento}

Se realizó la preparación del terreno (arada y rastrada), posteriormente se aplicó la fertilización inicial, un 40\% antes de la siembra y el 60\% distribuido en los cortes, previo al respectivo análisis de suelo, con las siguientes cantidades ( $\mathrm{kg} / \mathrm{ha} / \mathrm{año}$ ): Fertilización 1: Nitrato de Amonio 476 (34\%), Superfosfato Triple 285 (46\%) (80\% al inicio), Muriato de Potasio 135 (60\%) y 
Sulfato de Magnesio 107 (25\%). Para el fertiforraje costa establecimiento (13-26-13-4-3) se empleó 329 kg/ha al inicio y el fertiforraje costa producción (21-12-15-4-3) 493 kg/ha/ año en los cortes. Los fertilizantes orgánicos se incorporaron al voleo, un $60 \%$ a la siembra y un $40 \%$ distribuido en los cortes. Vermicompost bovino se empleó 24.7 t/ha y del Compost de bovino 26.6 t/ha. La siembra se realizó al voleo, empleándose $5 \mathrm{~kg} / \mathrm{ha}$ de semillas de pastos Mulato y Xaraés. El primer corte de igualación se efectuó a los 100 días después de la siembra, luego los cortes a los 30 días en invierno y 40 días en verano.

\section{Resultados y Discusión}

\subsection{Análisis combinado de los cuatro cortes}

En el ADEVA de las variables de los cuatro cortes Cuadro 1, se detectó una alta significación estadística en las cuatro variables para: El factor fertilización, CO1, CO2, cortes y para la interacción tratamientos $x$ cortes; también para pastos en las variables altura y materia verde; además de la interacción cortes $x$ repetición en las variables materia verde y seca. El coeficiente de variación (a) fue de 0.82\%, 7.2\%, 18.6\% y 18.6\%, respectivamente y el coeficiente de variación (b) fue de $2.14 \%$, $5.7 \%, 14.4 \%$ y $14.6 \%$, respectivamente para las cuatro variables; considerados aceptables. Los promedios generales de los cuatro cortes fueron de: $80.87 \%$ en cobertura, $86.6 \mathrm{~cm}$ en altura, $169.5 \mathrm{t} /$ ha/año en materia verde y 28.6 t/ha/año de materia seca.

En cuanto a los promedios de cobertura para fertilización, Figura 1, se observan dos rangos de significación, ubicándose en el primer rango las fertilizaciones químicas: f1 (fertilización 1) y f2 (fertiforraje) con $84.3 \%$ y $83.4 \%$ respectivamente, debido posiblemente a que los pastos responden rápidamente a la aplicación de minerales químicos, especialmente el nitrógeno, cuyo carácter de solubilidad y movilidad permite una rápida asimilación por parte de las plantas. En el segundo rango se ubicaron las fertilizaciones orgánicas: f3 (vermicompost) y f4 (compost) y el testigo sin fertilización (fO), presentando promedios de buena cobertura, pero inferior a los tratamientos con fertilización química, lo que implica que la fertilización química sí contribuye en el incremento de cobertura de los pastos brachiarias, evitando así la incidencia de malezas.

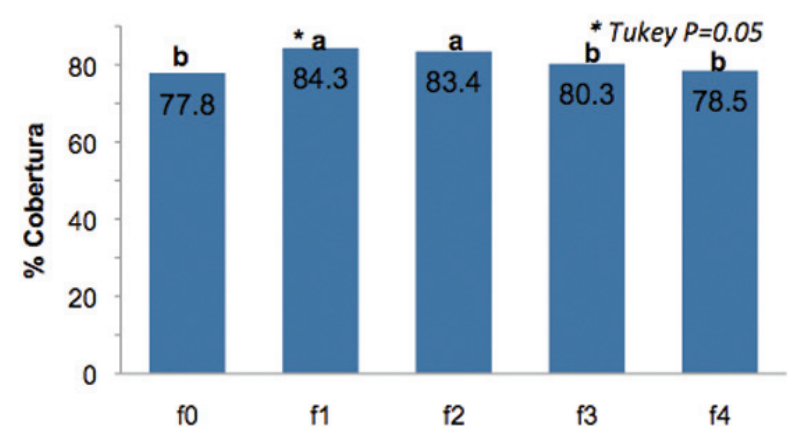

Figura 1. Promedios y prueba de Tukey al 5\% para cobertura (\%) de cinco fertilizaciones de Brachiaria (híbrida y brizantha Xaraés).

En cuanto a la altura de pastos Figura 2, el pasto Xaraés presentó el promedio más alto con $92.8 \mathrm{~cm}$ mientras que el pasto Mulato presentó una altura de $82.3 \mathrm{~cm}$. El pasto Xaraés posee mayor altura por tratarse de una variedad que desarrolla macollos más altos y erectos, con hojas largas, finas y sin pubescencia; en cambio el pasto Mulato presenta hojas de menor tamaño, más gruesas y con pubescencia (BURGOS, 2004).

Cuadro 1. Cuadrados Medios del ADEVA de Cobertura (\%), Altura (cm), Materia Verde (t/ha/año) y Materia Seca (t/ha/año) de la Fertilización Química y Órgano-mineral de Brachiaria (híbrida y brizantha Xaraés).

\begin{tabular}{|l|c|c|c|c|l|}
\hline \multirow{2}{*}{ Fuentes de } & \multirow{2}{*}{ Grados } & \multicolumn{4}{|l|}{ Cuadrados Medios } \\
\cline { 2 - 4 } & Libertad & Cobertura & Altura & M. Verde & M. Seca \\
Total & 159 & - & - & - & - \\
Repeticiones & 3 & $0.01 \mathrm{~ns}$ & $59.7 \mathrm{~ns}$ & $2997.7^{*}$ & $80.9 \mathrm{~ns}$ \\
Pastos & 1 & $0.00 \mathrm{~ns}$ & $6337.8^{* *}$ & $42050.9^{* *}$ & $14.6 \mathrm{~ns}$ \\
Fertilización & 4 & $0.21^{* *}$ & $947.9^{* *}$ & $19366.2^{* *}$ & $731.8^{* *}$ \\
CO1 (f0 vs f1,f2,f3 y f4) & 1 & $0.29^{* *}$ & $2112.7^{* *}$ & $39363.5^{* *}$ & $2078.3^{* *}$ \\
CO2 (f1,f2 vs f3,f4) & 1 & $0.50^{* *}$ & $1568.0^{* *}$ & $34195.5^{* *}$ & $643.9^{* *}$ \\
CO3 (f1 vs f2) & 1 & $0.01 \mathrm{~ns}$ & $11.7 \mathrm{~ns}$ & $3844.8 \mathrm{~ns}$ & $201.6^{*}$ \\
CO4 (f3 vs f4) & 1 & $0.04 \mathrm{~ns}$ & $99.5 \mathrm{~ns}$ & $61.0 \mathrm{~ns}$ & $3.3 \mathrm{~ns}$ \\
Pastos x Fertilización & 4 & $0.01 \mathrm{~ns}$ & $57.3 \mathrm{~ns}$ & $640.3 \mathrm{~ns}$ & $15.2 \mathrm{~ns}$ \\
Error (a) & 27 & 0.01 & 38.6 & 992.4 & 28.2 \\
Cortes & 3 & $3232.35^{* *}$ & $1710.2^{* *}$ & $12849.1^{* *}$ & $267.3^{* *}$ \\
Cortes x Repetición & 9 & $0.09 *$ & $44.7 \mathrm{~ns}$ & $1701.3^{* *}$ & $50.5^{* *}$ \\
Tratamientos x Cortes & 27 & $0.14 * *$ & $93.3^{* *}$ & $1363.9^{* *}$ & $49.5^{* *}$ \\
Error (b) & 81 & 0.04 & 24.1 & 595.1 & 17.3 \\
\hline Coeficiente de & & & & & \\
Variación(a) \% & - & 0.82 & 7.2 & 18.6 & 18.6 \\
Coeficiente de & & & & & \\
Variación (b)\% & - & 2.14 & 5.7 & 14.4 & 14.6 \\
Promedio & - & $80.87 \%$ & $86.6 \mathrm{~cm}$ & 169.5 t/ha/año & 28.6 t/ha/año \\
\hline
\end{tabular}




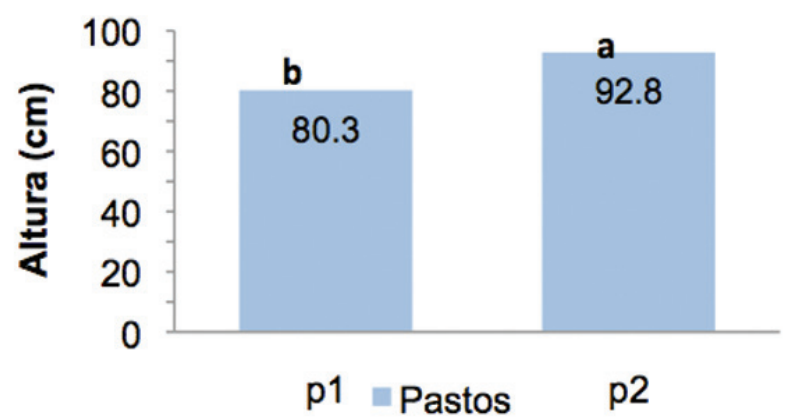

Figura 2. Promedios para altura $(\mathrm{cm})$ de dos pastos Brachiaria (híbrida y brizantha Xaraés).

En los promedios de altura para fertilización, Figura 3, se detectaron dos rangos de significación, ubicándose en el primer rango f1 (fertilización 1) con $92.3 \mathrm{~cm}$ y en último lugar del segundo rango se ubicó fo (sin fertilización) con $79.3 \mathrm{~cm}$; concluyéndose que la fertilización química y órgano-mineral si incidió en la altura del pasto, ya que los pastos mejorados absorben y utilizan el fertilizante más efectivamente que un pasto natural (NOBUI, 2004).

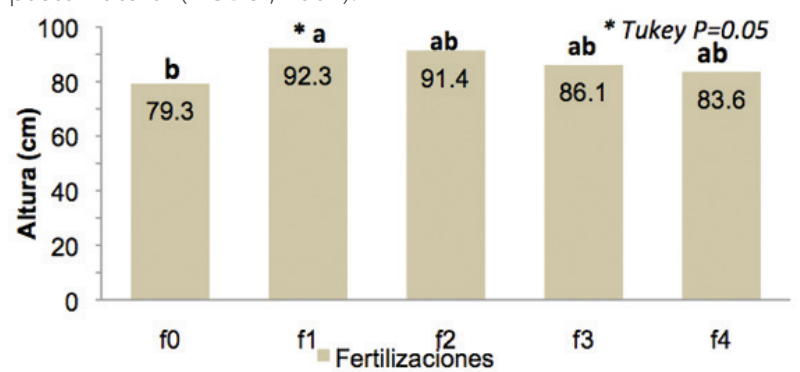

Figura 3. Promedios y Prueba de Tukey al $5 \%$ para altura $(\mathrm{cm})$ de cinco fertilizaciones de dos pastos Brachiaria (híbrida y brizantha Xaraés).

En los promedios para fertilización de materia verde, Figura 4, se detectaron dos rangos de significación, ubicándose en el primer rango la fertilización 1 (f1) con 201.6 t/ha/año y en último lugar del segundo rango se ubicó fo (sin fertilización) con 163.5 t/ha/año. Los tratamientos con fertilización tienen mayor producción de materia verde por una recuperación más rápida del potrero (LEÓN, 2003).

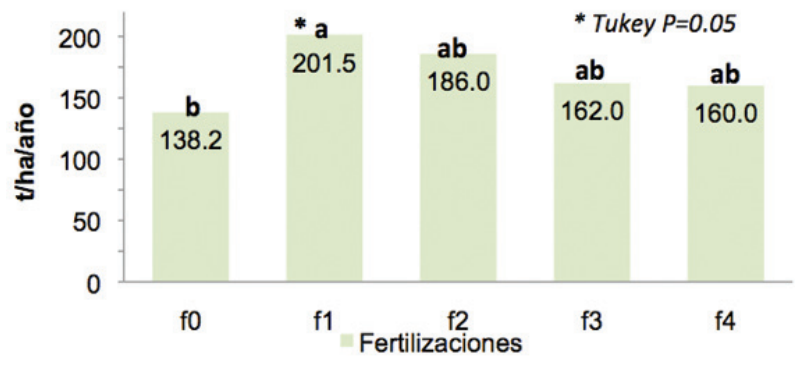

Figura 4. Promedios y Prueba de Tukey al $5 \%$ para la fertilización de la producción de materia verde de dos pastos Brachiaria.

En los promedios de rendimiento de materia seca para fertilización, Figura 5, se detectó dos rangos de significación estadística, ubicándose en el primer rango f1 (fertilización 1) con 34.4 t/ha/año y en último lugar del segundo rango fo (sin fertilización) con 21.4 t/ha/año, observándose que los tratamientos con la aplicación de fertilizantes tienen rendimientos superiores al tratamiento sin fertilizante (fO); El EMBRAPA (2004), manifiesta que el pasto Xaraés responde satisfactoriamente a la aplicación de dosis moderadas de cal dolomítica 2 a 4 t/ha y de fósforo 60 a 80 kg/ha.

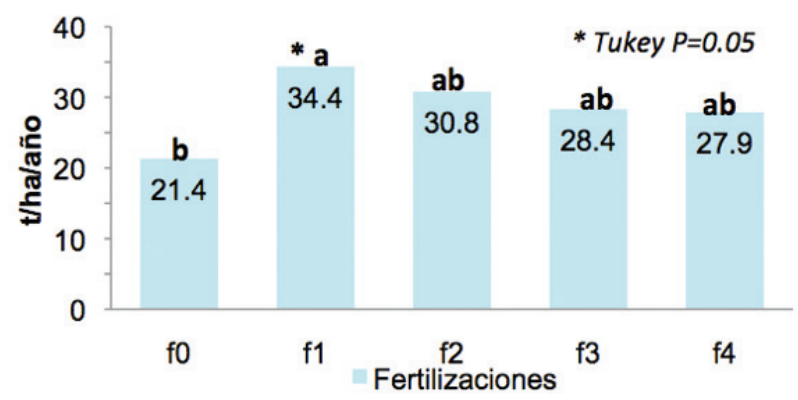

Figura 5. Promedios y Prueba de Tukey al 5\% para la fertilización del rendimiento de materia seca de dos pastos Brachiaria.

En los promedios de la TAMS para pastos, Figura 6, el pasto Xaraés (p2) se ubicó con mejor promedio con 18.4\%, seguido por el Mulato (p1) con 15.5\%; el pasto Mulato contiene menor cantidad de materia seca por presentar un alto contenido de humedad, pero su rendimiento de materia seca es superior al pasto Xaraés, debido a su elevada producción de materia verde. El CIAT, manifiesta que el pasto Mulato y la brizantha Libertad pueden presentar en época de lluvias una TAMS de 24\%, pudiendo incrementar estos valores en épocas de sequía a $28 \%$ para el Mulato y 31\% para el Xaraés, pero su contenido de proteína cruda se reduce de $9 \%$ a $8 \%$ en época seca (CIAT, 2004).

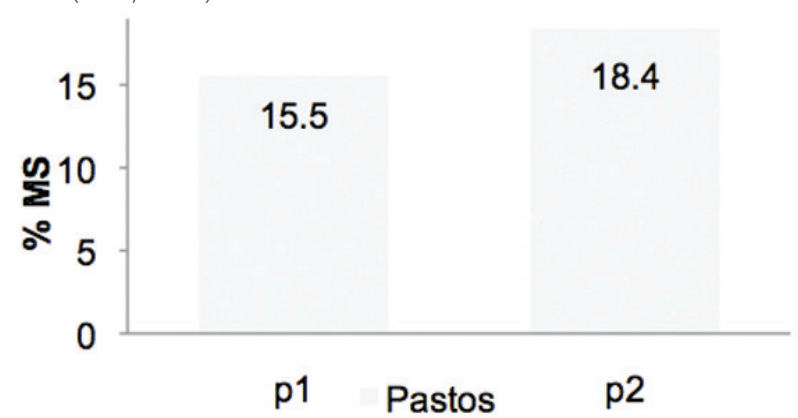

Figura 6. Promedios de la TAMS para dos pastos Brachiaria (híbrida y brizanthaXaraés).

En los promedios de la TAMS para la fertilización, Figura 7, el compost (f3) se ubicó con mejor promedio con $17.7 \%$, seguido por el vermicompost (f4) con $17.6 \%$ y al final sin fertilización (f0) con 15.5\%. Los tratamientos con abonos orgánicos producen mayor contenido de materia seca que los tratamientos con fertilizantes químicos, este resultado concuerda con lo manifestado por CHACON (1999), que sostiene que el vermicompost aumenta la resistencia de las plantas por su alto porcentaje de ácidos húmicos y elevado contenido de micro-elementos y la enorme carga bacteriana que posee. Los contenidos de los nutrimentos complementarios existentes en el vermicompost, contribuyen a la obtención de un mayor volumen de materia seca aprovechable. 


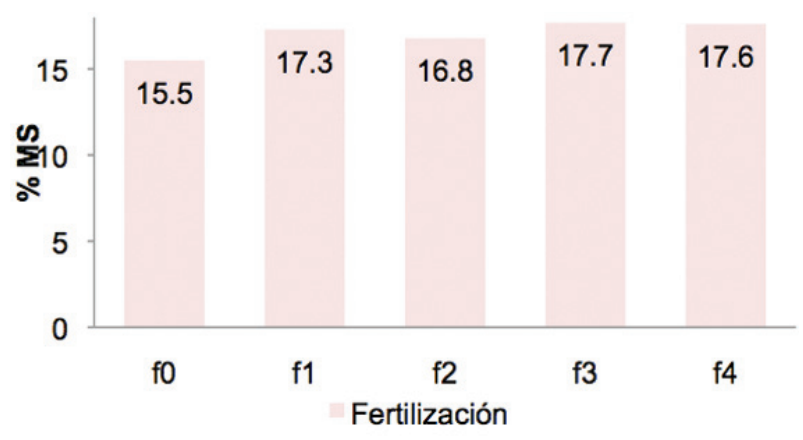

Figura 7. Promedios de la TAMS para la fertilización de dos pastos Brachiaria (híbrida y brizantha Xaraés).

\subsection{Análisis por épocas de corte \\ 3.2.1. Invierno}

Para la época de invierno se evaluaron tres cortes (Primer, segundo y cuarto corte), debido a las condiciones climáticas de esa época.

En el ADEVA de la época de invierno de las variables de tres cortes Cuadro 2, se detectó una alta significación estadística en las cuatro variables para: La fertilización, CO1, CO2, cortes y para la interacción tratamientos x cortes; también para pastos en las variables altura y materia verde; además de la interacción cortes $\mathrm{x}$ repetición en las variables materia verde y seca. El coeficiente de variación (a) fue de 1.0\%, 6.2\%, 18.1\% y $18.4 \%$, respectivamente y el coeficiente de variación (b) fue de $2.2 \%, 5.8 \%, 14.1 \%$ y $14.6 \%$, respectivamente para las cuatro variables; considerados aceptables. Los promedios generales de la época de invierno fueron de: $81.27 \%$ en cobertura, $88.2 \mathrm{~cm}$ en altura, 175.7 t/ha/año en materia verde y 29.1 t/ha/año de materia seca.

En los promedios de altura de pastos para épocas, Figura 8, se detectaron dos rangos de significación, ubicándose en el primer rango p2 (Xaraés) con $94.0 \mathrm{~cm}$ para invierno y $89.3 \mathrm{~cm}$ para verano, en el segundo rango se ubicó p1 (Mulato) con $82.4 \mathrm{~cm}$ y $73.7 \mathrm{~cm}$ respectivamente, para invierno y verano. El pasto Mulato alcanza un menor crecimiento por tratarse de un hibrido de crecimiento macollado de hábito decumbente capaz de enraizar a partir de los nudos cuando entran en estrecho contacto con el suelo (BURGOS, 2004). En cambio el pasto Xaraés alcanza una mayor altura, por tratarse de una variedad de rápido desarrollo con hojas de crecimiento erecto y por presentar una inflorescencia tardía, la cual beneficia al pasto para su mejor aprovechamiento por el ganado (MARANGATU, 2003).

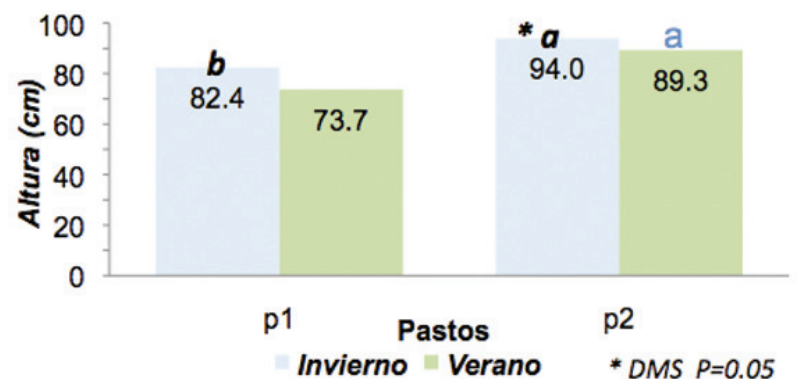

Figura 8. Promedios y Prueba de DMS al 5\% en épocas para altura (cm) de dos pastos Brachiaria (híbrida y brizantha Xaraés).

En los promedios para épocas de los pastos en la producción de materia verde, Figura 9, se observó en la época de invierno un solo rango de significación, ubicándose con

Cuadro 2. Cuadrados Medios del ADEVA de la Época de Invierno de Cobertura (\%), Altura (cm), Materia Verde (t/ha/año) y Materia Seca (t/hal año) de la Fertilización Química y Órgano-mineral de Brachiaria (híbrida y brizantha Xaraés).

\begin{tabular}{|c|c|c|c|c|c|}
\hline \multirow{2}{*}{$\begin{array}{l}\text { Fuentes de } \\
\text { Variación }\end{array}$} & \multirow{2}{*}{$\begin{array}{l}\text { Grados } \\
\text { Libertad }\end{array}$} & \multicolumn{4}{|c|}{ Cuadrados Medios } \\
\hline & & Cobertura & Altura & M. Verde & M. Seca \\
\hline Total & 119 & - & - & - & - \\
\hline Repeticiones & 3 & $0.02 \mathrm{~ns}$ & 98.9 ns & $2234.9 *$ & $60.6 \mathrm{~ns}$ \\
\hline Pastos & 1 & $0.00 \mathrm{~ns}$ & $837.2^{* *}$ & $26003.8 * *$ & $0.0 \mathrm{~ns}$ \\
\hline Fertilización & 4 & $0.17 * *$ & $4025.2 * *$ & $13110.3 * *$ & $491.7 * *$ \\
\hline $\mathrm{CO} 1$ (f0 vs f1,f2,f3 y f4) & 1 & $0.18 * *$ & $1466.5 * *$ & $22002.0^{* *}$ & $1105.1^{* *}$ \\
\hline $\mathrm{CO} 2(\mathrm{f} 1, \mathrm{f} 2$ vs $\mathrm{f} 3, \mathrm{f} 4)$ & 1 & $0.31 * *$ & $924.5 * *$ & $15088.2^{* *}$ & $248.3 * *$ \\
\hline $\mathrm{CO} 3$ (f1 vs f2) & 1 & $0.00 \mathrm{~ns}$ & $7.1 \mathrm{~ns}$ & 2195.9 ns & $121.7 *$ \\
\hline $\mathrm{CO} 4$ (f3 vs f4) & 1 & $0.03 \mathrm{~ns}$ & 46.9 ns & $43.9 \mathrm{~ns}$ & $0.1 \mathrm{~ns}$ \\
\hline Pastos x Fertilización & 4 & $0.01 \mathrm{~ns}$ & $62.4 \mathrm{~ns}$ & $373.8 \mathrm{~ns}$ & $6.6 \mathrm{~ns}$ \\
\hline Error (a) & 27 & 0.01 & 30.1 & 1010.4 & 28.7 \\
\hline Cortes & 2 & $3248.07 * *$ & $1885.5 * *$ & $10069.5^{* *}$ & $336.8 * *$ \\
\hline Cortes x Repetición & 6 & $0.09 *$ & $42.8 \mathrm{~ns}$ & $2120.7 * *$ & $61.4 * *$ \\
\hline Tratamientos $x$ Cortes & 18 & $0.14 * *$ & $114.1 * *$ & $1845.8^{* *}$ & $64.0 * *$ \\
\hline Error (b) & 54 & 0.04 & 25.7 & 637.6 & 17.9 \\
\hline $\begin{array}{l}\text { Coeficiente de } \\
\text { Variación(a) \% }\end{array}$ & - & 1.0 & 6.2 & 18.1 & 18.4 \\
\hline Coeficiente de & & & & & \\
\hline Variación (b)\% & - & 2.2 & 5.8 & 14.1 & 14.6 \\
\hline Promedio & - & $81.27 \%$ & $88.2 \mathrm{~cm}$ & 175.7 t/ha/año & 29.1 t/ha/año \\
\hline
\end{tabular}


mejor promedio el pasto Mulato (p1) con 190.4 t/ha/año, seguido por el pasto Xaraés (p2) con 161.0 t/ha/año. En la época de verano se detectaron dos rangos de significación, ubicándose en el primer rango p1 (Mulato) con 171.6 t/ha/año y en el segundo rango se ubicó el p2 (Xaraés) con 130.26 t/ha/

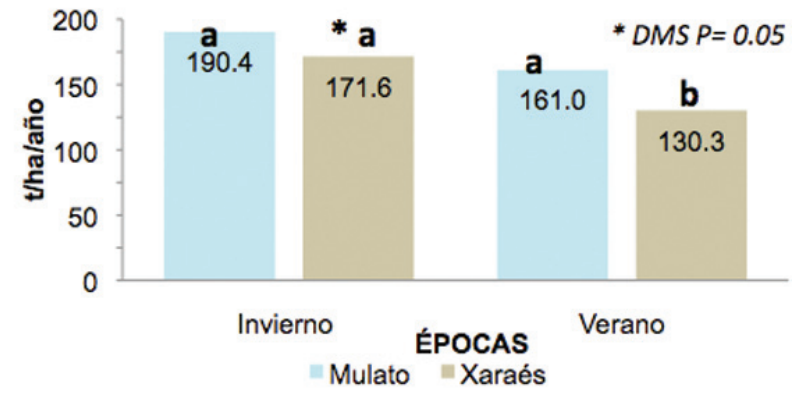

Figura 9. Promedios y Prueba de DMS al 5\% para épocas de dos pastos Brachiaria en la producción de materia verde.

Cuadro 3. Cuadrados Medios del ADEVA de la Época de Verano de Cobertura (\%), Altura (cm), Materia Verde (t/ha/año) y Materia Seca (t/ha/año) de la Fertilización Química y Órgano-mineral de Brachiaria (híbrida y brizantha Xaraés).

\subsubsection{Verano}

En el ADEVA de la época de verano de las variables del tercer corte Cuadro 3, se detectó una alta significación estadística para la variable cobertura en: fertilización, CO1 y CO2; para las variables altura y materia verde en: fertilización, CO1, CO2 y CO3; en materia seca en la CO1 y CO2. El coeficiente de variación fue de $1.8 \%, 6.6 \%, 14.7 \%$ y $14.6 \%$, respectivamente para las cuatro variables; considerados aceptables. El promedio general de la época de verano fue de: $79.69 \%$ en cobertura, 81.5 cm en altura, 150.9 t/ha/año en materia verde y 27.0 t/ha/año de materia seca.

\subsection{Tratamientos Adicionales}

En el ADEVA de los tratamientos adicionales de las cuatro variables Cuadro 4, se detectó una alta significación estadística la variable cobertura en pastos, para la variable altura en: pastos, fertilización y CO1; en materia seca para la CO1. El coeficiente de variación fue de 2.25\%, 5.2\%, 19.1\% y 20.1\%, respectivamente para las cuatro variables. El promedio general de los tratamientos adicionales fueron de: $75.0 \%$ en cobertura, $83.5 \mathrm{~cm}$ en altura, $161.5 \mathrm{t} / \mathrm{ha} / \mathrm{año}$ en materia verde y $27.1 \mathrm{t} / \mathrm{ha} /$ año de materia seca.

\begin{tabular}{|c|c|c|c|c|c|}
\hline \multirow{2}{*}{$\begin{array}{l}\text { Fuentes de } \\
\text { Variación }\end{array}$} & \multirow{2}{*}{$\begin{array}{l}\text { Grados } \\
\text { Libertad }\end{array}$} & \multicolumn{4}{|c|}{ Cuadrados Medios } \\
\hline & & Cobertura & Altura & M. Verde & M. Seca \\
\hline Total & 39 & - & - & - & - \\
\hline Pastos & 1 & $0.00 \mathrm{~ns}$ & $9.3 \mathrm{~ns}$ & $1625.2 *$ & $49.3 *$ \\
\hline Fertilización & 4 & $0.35 * *$ & $2433.6 * *$ & $17114.2 * *$ & $54.3 \mathrm{~ns}$ \\
\hline CO1 (f0 vs f1,f2,f3 y f4) & 1 & $0.46 * *$ & $153.7^{* *}$ & $6592.4^{* *}$ & $257.0 *$ \\
\hline $\mathrm{CO} 2(\mathrm{f} 1, \mathrm{f} 2$ vs f3,f4) & 1 & $0.83 * *$ & $924.5 * *$ & $10027.4^{* *}$ & $609.6 * *$ \\
\hline CO3 (f1 vs f2) & 1 & $0.06 \mathrm{~ns}$ & $338.0 * *$ & $15418.8 * *$ & $370.1 * *$ \\
\hline CO4 (f3 vs f4) & 1 & $0.04 \mathrm{~ns}$ & $2.4 \mathrm{~ns}$ & 917.7 ns & $40.1 \mathrm{~ns}$ \\
\hline Pastos x Fertilización & 4 & $0.05 \mathrm{~ns}$ & $39.1 \mathrm{~ns}$ & $5.6 \mathrm{~ns}$ & $8.3 \mathrm{~ns}$ \\
\hline Repeticiones & 3 & $0.09 *$ & $60.1 \mathrm{~ns}$ & $536.6 \mathrm{~ns}$ & $27.5 \mathrm{~ns}$ \\
\hline Error experimental & 27 & 0.03 & 29.3 & 491.9 & 15.4 \\
\hline Coeficiente de & & & & & \\
\hline Variación(\%) & - & 1.8 & 6.6 & 14.7 & 14.6 \\
\hline Promedio & - & $79.69 \%$ & $81.5 \mathrm{~cm}$ & 150.9 t/ha/año & 27.0 t/ha/año \\
\hline
\end{tabular}

Cuadro 4. Cuadrados Medios del ADEVA de los Tratamientos Adicionales de Cobertura (\%), Altura (cm), Materia Verde (t/ha/año) y Materia Seca (t/ha/año) de la Fertilización Química y Órgano-mineral de Brachiaria (híbrida y brizantha Xaraés).

\begin{tabular}{|l|c|c|c|c|c|}
\hline \multirow{2}{*}{ Fuentes de } & \multirow{2}{*}{ Grados } & \multicolumn{4}{|l|}{ Cuadrados Medios } \\
\cline { 2 - 5 } Variación & Libertad & Cobertura & Altura & M. Verde & M. Seca \\
Total & 31 & - & - & - & - \\
Pastos & 1 & $1.0^{* *}$ & $844,6^{* *}$ & $5471.3 \mathrm{~ns}$ & $1.0 \mathrm{~ns}$ \\
Fertilización & 3 & $0.01 \mathrm{~ns}$ & $303.9^{* *}$ & $664.2 \mathrm{~ns}$ & $72.2 \mathrm{~ns}$ \\
CO1 (t0 vs t1,t2 y t3) & 1 & $0.00 \mathrm{~ns}$ & $878.5^{* *}$ & $1694.1 \mathrm{~ns}$ & $191.4^{* *}$ \\
CO2 (t1 vs t2,t3) & 1 & $0.01 \mathrm{~ns}$ & $13.2 \mathrm{~ns}$ & $296.4 \mathrm{~ns}$ & $14.7 \mathrm{~ns}$ \\
CO3 t2 vs t3) & 1 & $0.02 \mathrm{~ns}$ & $20.2 \mathrm{~ns}$ & $2.0 \mathrm{~ns}$ & $10.5 \mathrm{~ns}$ \\
Pastos x Fertilización & 3 & $0.25^{* *}$ & $58.3 \mathrm{~ns}$ & $1996.2 \mathrm{~ns}$ & $73.9 \mathrm{~ns}$ \\
Repeticiones & 3 & $0.32^{* *}$ & $262.2 * *$ & $1585.2 \mathrm{~ns}$ & $55.5 \mathrm{~ns}$ \\
Error experimental & 21 & 0.04 & 18.8 & 954.4 & 29.7 \\
Coeficiente de & - & 2.25 & & & \\
Variación(\%) & - & $75.00 \%$ & $83.5 \mathrm{~cm}$ & 161.5 t/ha/año & 27.1 t/ha/año \\
Promedio & - & &
\end{tabular}




\subsection{Calidad del forraje}

\subsubsection{Análisis Bromatológicos}

En los promedios de los análisis bromatológicos Cuadro 5, para humedad se observa con menor promedio al T10 (Xaraés con compost) con 81.4\%; mientras más bajo es el contenido de humedad mejor es el pasto, por contener mayor cantidad de materia seca.

Para proteína se ubicó con mejor promedio el T2 (Mulato con fertilización 1) con 20.8\%, seguido por T4 (Mulato con vermicompost) con $18.6 \%$, en los últimos lugares se ubicaron los testigos T1 (Mulato sin fertilización) y T6 (Xaraés sin fertilización) con $16.4 \%$ y $16.4 \%$, respectivamente. Lo que concuerda con BURGOS (2004), que manifiesta que el pasto Mulato presenta porcentajes de proteína que alcanzan entre 12 y $16 \%$ con una digestibilidad de hasta 62\%; el EMBRAPA (2004), manifiesta que el pasto Xaraés a las cinco semanas de rebrote obtiene un 62\% de DIVMS y de proteína bruta un 8.5\%.

En el porcentaje de grasa (extracto etéreo), el T10 (Xaraés con compost) se ubicó con mejor promedio con 13.3\%. El contenido de lípidos varia entre 3 y $10 \%$ y generalmente declina con la edad del pasto (BERNAL, J. 1994). El porcentaje más bajo de ceniza se encuentra en elT7 (Xaraés con fertilización 1) con 10.2\%. El contenido más alto de fibra lo obtiene el T10 (Xaraés con compost) con $27.8 \%$ de fibra, de los constituyentes de las paredes celulares del pasto.

\subsubsection{Análisis Foliares}

En los promedios de los análisis foliares de los tratamientos, Cuadro 6, para nitrógeno se observó que el nivel más alto lo presentó el T2 (Mulato con fertilización 1) con $3.2 \%$, considerado como un nivel medio. Para el fósforo el nivel más alto lo presentó el T5 (Mulato con compost) con 0.28\%, en general todos los promedios a excepción de los testigos (T1 y T6) tienen una concentración media de fósforo. En el potasio se observó el mayor contenido en el T5 (Mulato con compost) y en el T4 (Mulato con vermicompost) con 4.5\% y $4.4 \%$, respectivamente, siendo de un nivel alto, según INPOFOS (2003); todos los tratamientos presentan un alto contenido de potasio en al análisis foliar, posiblemente se debe a que el género Brachiaria absorbe cantidades de potasio superiores a las de nitrógeno, por lo que sus hojas presentan un alto contenido del mismo. El nivel más alto de calcio lo alcanzó el T2 (Mulato con fertilización 1) con 1.11\%; en los niveles más altos de magnesio se ubicaron el T2 (Mulato con fertilización 1), el T7 (Xaraés con fertilización1) y el T8 (Xaraés con fertiforraje) con 0.36\%; para el azufre el valor más alto se obtuvo en el T2 (Mulato con fertilización 1) y en el T3 (Mulato con fertiforraje) con $0.16 \%$. Estos resultados del análisis foliar de la cantidad de nutrientes en la materia seca de los pastos son mucho más altos que los manifestados por BERNAL, J. (1994), a los 35 días después del corte en la prefloración.

Cuadro 5. Promedios de los análisis bromatológicos para tratamientos de dos pastos Brachiaria (híbrida y brizantha Xaraés).

\begin{tabular}{|l|l|l|l|l|l|l|}
\hline Tratamiento & Descripción & Humedad \% & Proteína \% & Grasa \% & Ceniza \% & Fibra \% \\
T1 & p1f0 & 86.2 & 16.4 & 8.7 & 11.7 & 23.1 \\
T2 & p1f1 & 85.4 & 20.8 & 8.7 & 12.9 & 23.6 \\
T3 & p1f2 & 85.7 & 17.8 & 8.1 & 13.2 & 23.3 \\
T4 & p1f3 & 84.4 & 18.6 & 8.6 & 12.9 & 23.6 \\
T5 & p1f4 & 84.5 & 17.7 & 9.0 & 12.2 & 23.7 \\
T6 & p2f0 & 84.2 & 16.4 & 8.6 & 11.0 & 22.9 \\
T7 & p2f1 & 81.5 & 17.7 & 9.4 & 10.2 & 28.1 \\
T8 & p2f2 & 82.8 & 17.5 & 8.9 & 10.3 & 26.8 \\
T9 & p2f3 & 81.9 & 17.4 & 10.9 & 10.3 & 26.4 \\
T10 & p2f4 & 81.4 & 17.2 & 13.3 & 10.6 & 27.8 \\
\hline
\end{tabular}


Cuadro 6. Promedios de análisis foliares para tratamientos de dos pastos Brachiaria (hibrida y brizantha Xaraés).

\begin{tabular}{|l|l|l|l|l|l|l|l|l|l|l|l|l|}
\hline Trata- & Descrip- & $\mathrm{N}$ & $\mathrm{P}$ & $\mathrm{K}$ & $\mathrm{Ca}$ & $\mathrm{Mg}$ & $\mathrm{S}$ & $\mathrm{Cu}$ & $\mathrm{B}$ & $\mathrm{Fe}$ & $\mathrm{Zn}$ & $\mathrm{Mn}$ \\
miento & ción & $\%$ & $\%$ & $\%$ & $\%$ & $\%$ & $\%$ & $\mathrm{ppm}$ & $\mathrm{ppm}$ & $\mathrm{ppm}$ & $\mathrm{ppm}$ & $\mathrm{ppm}$ \\
T1 & p1f0 & 2.30 & 0.17 & 3.8 & 1.07 & 0.26 & 0.12 & 9.0 & 0.45 & 124 & 17 & 24 \\
T2 & p1f1 & 3.17 & 0.26 & 3.8 & 1.11 & 0.36 & 0.16 & 8.5 & 9.80 & 178 & 36 & 34 \\
T3 & p1f2 & 2.97 & 0.25 & 3.9 & 0.98 & 0.31 & 0.16 & 8.5 & 9.75 & 104 & 36 & 44 \\
T4 & p1f3 & 2.48 & 0.23 & 4.4 & 0.96 & 0.29 & 0.14 & 11.0 & 9.25 & 98 & 41 & 29 \\
T5 & p1f4 & 2.75 & 0.28 & 4.5 & 0.88 & 0.31 & 0.15 & 10.0 & 15.75 & 119 & 39 & 38 \\
T6 & p2f0 & 2.27 & 0.17 & 3.4 & 0.82 & 0.28 & 0.11 & 9.5 & 1.90 & 117 & 31 & 38 \\
T7 & p2f1 & 2.59 & 0.24 & 3.5 & 0.88 & 0.36 & 0.14 & 14.0 & 7.50 & 139 & 38 & 60 \\
T8 & p2f2 & 2.39 & 0.25 & 3.3 & 0.84 & 0.36 & 0.11 & 7.0 & 6.00 & 144 & 34 & 53 \\
T9 & p2f3 & 2.52 & 0.22 & 3.7 & 0.78 & 0.30 & 0.11 & 7.5 & 8.25 & 105 & 37 & 43 \\
T10 & p2f4 & 2.47 & 0.23 & 3.9 & 0.76 & 0.28 & 0.11 & 9.0 & 8.75 & 122 & 35 & 52 \\
\hline
\end{tabular}

\subsubsection{Análisis de Suelos}

En los promedios de los tratamientos del análisis del suelo al final de la investigación, Cuadro 7, se observó el mayor contenido de materia orgánica en el T4 (Mulato con vermicompost) con $1546 \mathrm{~kg} / \mathrm{ha} / \mathrm{año}$, concuerda con lo expresado por INPOFOS (2003), que al utilizar solamente abonos orgánicos como fuente de nutrientes se pierde de vista el mayor efecto benéfico de estos que es la acumulación de materia orgánica en el suelo, para el mantenimiento de la fertilidad y la sostenibilidad de la productividad del suelo.

En el contenido de nitrógeno, el T7 (Xaraés con fertilización 1) se ubicó primero con 31.1 kg/ha/año, debido a que el pasto Xaraés absorbe menos nitrógeno que el Mulato; el mayor contenido de fósforo, lo obtuvo el T3 (Mulato con fertiforraje) con $30.5 \mathrm{~kg} / \mathrm{ha} /$ año, posiblemente se debe a que el fertiforraje tiene una fórmula compuesta (21-12-15-4-3) en la que se aplica $43 \mathrm{~kg} / \mathrm{ha}$ de exceso de fósforo; para el potasio, se observó el mayor contenido en el T2 (Mulato con fertilización 1) con $343.2 \mathrm{~kg} / \mathrm{ha} / \mathrm{año}$, muy superior a los otros tratamientos. En el contenido de calcio, se ubicó primero el T4 (Mulato con vermicompost) con 2800 kg/ha/año; para magnesio el más alto contenido lo presentaron el T2 (Mulato con fertilización 1), el T5 (Mulato con compost) y el T9 (Xaraés con vermicompost) con 264 kg/ha/año; en boro el más alto contenido lo presentó el T5 (Mulato con compost) con 0.18 kg/ha/año. En el cobre se observó el más alto contenido en el T3 (Mulato con fertiforraje) con 18.26 kg/ha/año; el T7 (Xaraés con fertilización 1) con 682 kg/ha/año se ubicó con mayor contenido de hierro; en el contenido de manganeso se ubicó primero el T6 (Xaraés sin fertilización) con 9.06 kg/ha/año; y el T2 (Mulato con fertilización 1) se ubicó primero con $39.4 \mathrm{~kg} / \mathrm{ha} / \mathrm{año}$ en el contenido de zinc.

Cuadro 7. Resultados finales de los análisis de suelos para los tratamientos de dos pastos Brachiaria (híbrida y brizantha Xaraés).

\begin{tabular}{|c|c|c|c|c|c|c|c|c|c|c|c|c|c|c|}
\hline Tratamiento & Descripción & $\mathrm{pH}$ & CE ds/m & MO & $\mathrm{N}$ & $P$ & K & $\mathrm{Ca}$ & $|\mathrm{Mg}|$ & & $\mathrm{Cu}$ & $\mathrm{Fe}$ & $\mathrm{Mn}$ & $\mathrm{Zn}$ \\
\hline & & & & \multicolumn{11}{|c|}{ kg/ha/año } \\
\hline T1 & p1f0 & 5.8 & 0.24 & 940 & 28.1 & 10.0 & 101.4 & 2000 & 168 & 0.02 & 15.1 & 558 & 8.3 & 23.8 \\
\hline T2 & p1f1 & 5.9 & 0.25 & 1256 & 29.4 & 9.7 & 343.2 & 2400 & 264 & 0.06 & 8.9 & 676 & 7.5 & 39.4 \\
\hline T3 & $\mathrm{p} 1 \mathrm{f} 2$ & 5.8 & 0.28 & 1076 & 23.0 & 30.5 & 132.6 & 2000 & 216 & 0.02 & 18.3 & 668 & 7.2 & 28.8 \\
\hline T4 & p1f3 & 6.0 & 0.32 & 1546 & 30.1 & 12.7 & 132.6 & 2800 & 240 & 0.06 & 14.0 & 658 & 5.6 & 29.8 \\
\hline T5 & $\mathrm{p} 1 \mathrm{f} 4$ & 6.0 & 0.22 & 1382 & 30.1 & 14.1 & 132.6 & 2400 & 264 & 0.18 & 15.5 & 650 & 5.0 & 22.8 \\
\hline T6 & $\mathrm{p} 2 \mathrm{fO}$ & 5.9 & 0.24 & 1008 & 27.5 & 11.2 & 124.8 & 1960 & 180 & 0.02 & 17.5 & 594 & 9.1 & 24.2 \\
\hline T7 & $\mathrm{p} 2 \mathrm{f} 1$ & 5.8 & 0.30 & 1344 & 31.1 & 21.2 & 265.2 & 2400 & 216 & 0.02 & 15.5 & 682 & 8.4 & 28.4 \\
\hline T8 & $\mathrm{p} 2 \mathrm{f} 2$ & 5.7 & 0.28 & 1276 & 28.1 & 25.3 & 117.0 & 2000 & 240 & 0.02 & 15.5 & 646 & 7.2 & 23.4 \\
\hline T9 & $\mathrm{p} 2 \mathrm{f3}$ & 5.9 & 0.24 & 1412 & 28.1 & 13.4 & 124.8 & 2400 & 264 & 0.02 & 17.5 & 666 & 8.1 & 28.2 \\
\hline T10 & $\mathrm{p} 2 \mathrm{f} 4$ & 6.0 & 0.24 & 1412 & 27.8 & 13.0 & 117.0 & 2000 & 216 & 0.04 & 16.3 & 664 & 8.0 & 25.4 \\
\hline T11 & Maní & 5.9 & 0.14 & 1004 & 20.0 & 9.7 & 93.6 & 1600 & 144 & 0.04 & 18.3 & 570 & 8.4 & 23.0 \\
\hline
\end{tabular}




\subsection{Análisis Económico}

En el presupuesto parcial y análisis de dominancia de los tratamientos, Cuadro 8, se observó con mayor beneficio neto al T7 (Xaraés con fertilización 1) con 388 dólares/ha/año, seguido por el T2 (Mulato con fertilización 1) con 366 dólares/ ha/año, el pasto Xaraés alcanza el mejor beneficio neto, debido al costo más bajo de la semilla por ser una variedad y no un híbrido como el Mulato, ya que los rendimientos de materia seca son similares, de 28.86 y 28.26 t/ha/año, para Mulato y Xaraés, respectivamente.

El costo de producción más bajo lo presentó el T6 (Xaraés sin fertilización) con 526 dólares/ha/año, seguido por el T1 (Mulato sin fertilización) con 674 dólares/ha/año, debido a que los costos variables de los pastos son menores por tratarse de los testigos sin fertilización y el T2 (Mulato con fertilización
1) presentó el costo de producción más alto con 1135 dólares. En el análisis funcional de los tratamientos no dominados (T7 y T6), se detectó con mejor Tasa de Retorno Marginal (TRM) al T7 (Xaraés con fertilización 1) con 46.6\%, lo que significa que por cada dólar invertido se recupera 0.47 dólares, en comparación con el testigo T6 (Xaraés sin fertilización).

En el análisis económico del rendimiento y la relación beneficio/costo, Cuadro 9, se observó que la relación Beneficio/ Costo más alta se encontró en el T6 (Xaraés sin fertilización) con 1.44, que constituye el testigo con el pasto Xaraés, pero su calidad de pasto es inferior a la de los tratamientos con fertilización química, ubicándose el T8 (Xaraés con fertiforraje) y el T7 (Xaraés con fertilización 1) como la mejor opción de la relación $\mathrm{B} / \mathrm{C}$ para las fertilizaciones de estos pastos.

Cuadro 8. Presupuesto parcial y análisis de dominancia de los tratamientos dos pastos Brachiaria (híbrida y brizantha Xaraés).

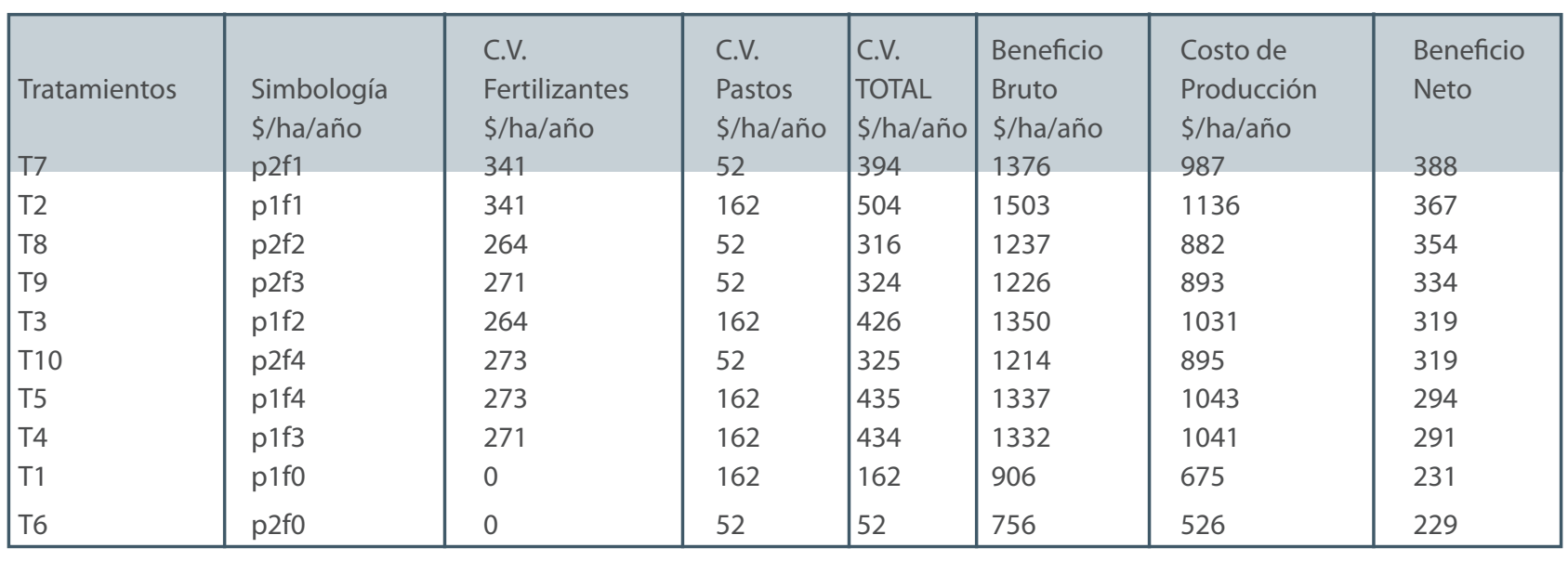

Cuadro 9. Análisis económico del rendimiento y beneficio/costo de los tratamientos para dos pastos Brachiaria (híbrida y brizantha Xaraés).

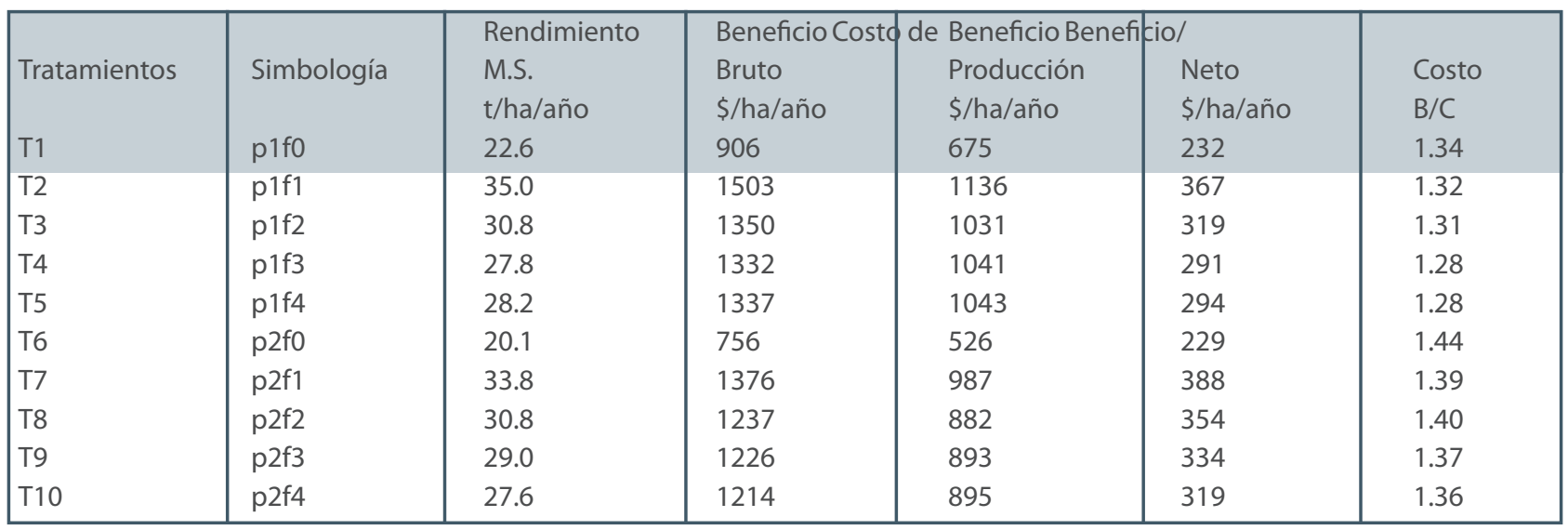




\section{Conclusiones}

- El mejor rendimiento de materia seca, la obtuvo el pasto Mulato (Brachiaria hibrido) con 35.0 t/ha/año, seguido por el pasto Xaraés (Brachiaria brizantha Xaraés) con 33.8 t/ha/año, sometidos en ambos casos a la fertilización 1.

- La concentración más alta de proteína con 20.8\% del análisis bromatológico y los mejores niveles de contenido de nutrientes en el análisis foliar lo presentó el T2 (Mulato con fertilización 1).

- La Tasa de Acumulación de Materia Seca más alta la consiguió el T9 (Xaraés con vermicompost) con 19.2\%.

- El mejor porcentaje de cobertura de Brachiaria se observó en el T2 (Mulato con fertilización 1) con 85.6\%.

- La mayor altura de pasto Xaraés se obtuvo con el T7 (Xaraés con fertilización 1) con $99.6 \mathrm{~cm}$ y del pasto Mulato en el T3 (Mulato con fertiforraje) con $85.1 \mathrm{~cm}$.

- Los suelos sometidos a la fertilización orgánica resultaron mejorados significativamente en términos porcentuales de materia orgánica y provisión de nutrimentos (nitrógeno, calcio, magnesio y boro) al final del ensayo.

- Desde el punto de vista económico, con el T7 (Xaraés con fertilización 1), se consiguió el más alto beneficio neto con 388 dólares/ha/año, seguido por el T2 (Mulato con fertilización 1) con 367 dólares/ha/año.

- La mejor Tasa de Retorno Marginal la presentó el T7 (Xaraés con fertilización 1) con 46.6\%, la relación Beneficio/Costo más alta se la obtuvo con el T6 (Xaraés sin fertilización) con 1.44, seguida por el T8 (Xaraés con fertiforraje) con 1.40 con la que se obtiene mejor calidad del pasto.

\section{Recomendaciones}

Para las condiciones agroecológicas del área del ensayo y sectores con similares características, se recomienda:

- Fertilizar los pastos Mulato (Brachiaria hibrido) y Xaraés (Brachiaria brizantha Xaraés) con la Fertilización 1 (Mezcla de fertilizantes químicos simples) en cantidad de 1026 kg/ha/año. - Aplicar Compost o Vermicompost a los pastos Mulato (Brachiaria hibrido) y Xaraés (Brachiaria brizantha Xaraés) en cantidad de $26.6 \mathrm{t} / \mathrm{ha}$ y $24.7 \mathrm{t} / \mathrm{ha}$, respectivamente, con una frecuencia de cada tres años.

- Para futuras investigaciones, asociar los pastos de este ensayo con leguminosas arbustivas para mejorar la calidad nutrimental del pastizal.

\section{Bibliografía}

AGRIPAC. (2004). Boletín divulgativo. Pasto Mulato (Brachiaria hibrido). Guayaquil- Ecuador, 6p.

ARGEL, P; LOBO, M; SANDOVAL, B. y MESEN, M. (2002). Documento Pasto Mulato. (Brachiaria híbrido, CIAT 36061). Costa Rica. Pp. 1-26.

BERNAL, J. (1994). Pastos y Forrajes Tropicales. Producción y Manejo. 3ra edición. Bogotá, Colombia. Pp. 22-23.

BURGOS, C. (2004). Pasto Mulato, Brachiaria Hibrido (CIAT 36061). Dirección de Ciencia y Tecnología Agropecuaria (DICTA), de la Secretaría de Agricultura y Ganadería (SAG). Tegucigalpa, Honduras. (En línea). Disponible en: http://www.sag.gob.hn/ pdf/Mulato.

Centro Internacional de Agricultura Tropical (CIAT). (1982). Manual para la Evaluación Agronómica. Red Internacional de Evaluación de Pastos Tropicales. Cali, Colombia. 170p.

CIAT. (2001). Reporte anual 2001. Convenio CIAT - Semillas Papalotla S.A. Pastos Tropicales y Leguminosas. Cali, Colombia. Pp.110-112.

CIAT. (2002). Mulato, Brachiaria Hibrido (CIAT 36061). Dirección de Ciencia y Tecnología Agropecuaria (DICTA), Secretaría de Agricultura y Ganadería (SAG). Tegucigalpa, Honduras. Disponible en: htp:/www.grupopapalotla.com.

- CIAT. (2003). Reporte anual 2003. Pastos Tropicales y Leguminosas. Cali, Colombia. 132p.

- Centro Internacional de Mejoramiento de maíz y Trigo (CIMMYT). (1988). Formulación y recomendaciones a partir de datos agronómicos: Manual metodológico de evaluación económica. Folleto de información № 27.

- Empresa Brasileira de Pesquisa Agropecuaria (EMBRAPA). (2004). Capim-Xaraés (Brachiaria brizantha cv. Xaraés). Brasil. (En línea).. Disponible en: www.cpafro.embrapabr/embrapa bases/Xaraés. htm.

· Fertilizantes e Insumos S.A. (FERTISA). (2003). Boletín divulgativo. Fertilizantes: Fertiforraje Costa. Guayaquil - Ecuador. 2p.

FERTISA. (2004). Boletín divulgativo. Pasto Xaraés (Brachiaria brizantha Xaraés). Guayaquil-Ecuador. 2p.

GUIOT, G. y MELENDEZ, N. (2002). Comparación morfológica de Brachiaria híbrido cv. Mulato y Brachiaria brizantha cv. Insurgente. XV Reunión Científica Tecnológica Forestal y Agropecuaria. Tabasco, México.

- INIAP. (2005). Datos Meteorológicos de Temperatura, Precipitación, Humedad Relativa y Heliofania de los años 20002004. Estación Experimental Santo Domingo - Ecuador. 4p. - INPOFOS. (2003). Manual de Nutrición y Fertilización de Pastos. Quito - Ecuador. 94p.

- JACOME, L. (1997). Tesis de Control de Salivazo (Aeneolamia sp.) en pasto Brachiaria (Brachiaria decumbens). Universidad Central. Facultad de Ciencias Agrícolas. Quito - Ecuador. 123p. 
- Ministerio de Agricultura y Ganadería (MAG). (2003). Agencia de Servicios Agropecuarios. Distribución de los Pastizales en el Cantón Santo Domingo - Ecuador. Pp. 3-6.

- MARANGATU SEMENTES. (2003). Cultivar Xaraés. Boletín Divulgativo. Ribeirao Preto, Brasil. 3p.

- MELENDEZ, N. F. (2003). Evaluación agronómica de tres pastos bajo pastoreo en dos localidades del trópico mexicano. INIFAP - CIR - Golfo - Centro. Informe Técnico. Convenio INIFAP Semillas Papalotla S.A. de C. V.

- MG5 XARAES. (2005). Ficha Técnica de Brizantha Xaraés. Perú. (En línea). Disponible en: www.huallamayo.com.pe/bbxoraes. htm. 3p.

- SILVA, E. (2004). Bioestadística y Diseño Experimental. Diseño de Bloques Completos al Azar. Universidad Tecnológica Equinoccial. Maestría de Nutrición Vegetal. Santo DomingoEcuador.

- SUQUILANDA, M. (2006). Elaboración, Uso y Manejo de los Abonos Orgánicos. Universidad Tecnológica Equinoccial. Maestría de Nutrición Vegetal. Santo Domingo - Ecuador. 